Service social

\title{
Les valeurs des cégépiens et la réussite scolaire : portrait des valeurs et repères pour l'intervention
}

\section{Jacques Roy}

Volume 52, numéro 1, 2006

URI : https://id.erudit.org/iderudit/015953ar

DOI : https://doi.org/10.7202/015953ar

Aller au sommaire du numéro

Éditeur(s)

École de service social de l'Université Laval

ISSN

1708-1734 (numérique)

Découvrir la revue

Citer cet article

Roy, J. (2006). Les valeurs des cégépiens et la réussite scolaire : portrait des valeurs et repères pour l'intervention. Service social, 52(1), 31-46.

https://doi.org/10.7202/015953ar
Résumé de l'article

L'article fait état des principaux résultats d'une recherche portant sur la réussite scolaire en milieu collégial. Plus spécifiquement, nous y traitons d'un des volets de l'étude, celui de l'influence du système de valeurs des cégépiens sur leur trajectoire scolaire. La recherche a été menée auprès d'un échantillon aléatoire de 744 étudiants de trois cégeps (Gaspésie et des Îles, Sainte-Foy, Vieux-Montréal). Un portrait des valeurs des cégépiens en relation avec la réussite scolaire est reproduit. Une comparaison avec d'autres études (québécoises, canadiennes et européennes) est faite au besoin pour mieux contextualiser les résultats de notre recherche. Nous nous appliquons de plus à évaluer l'importance que revêt le système de valeurs des cégépiens pour la réussite scolaire, tant sur le plan du rendement scolaire que sur celui de la persévérance aux études. À cet égard, les valeurs des étudiants figurent parmi les premiers déterminants de la réussite scolaire selon nos travaux. Enfin, l'article propose quelques paramètres d'intervention visant à favoriser la réussite scolaire. Ces paramètres ont été conçus sur la base des résultats de la recherche et ils incorporent des facteurs sociaux tenant à la réalité des cégépiens dont, au premier chef, leur système de valeurs. 


\title{
Les valeurs des cégépiens et la réussite scolaire : portrait des valeurs et repères pour l'intervention
}

\author{
Jacques RoY \\ Professeur-chercheur \\ Cégep de Sainte-Foy \\ Membre-chercheur \\ Observatoire Jeunes et Société
}

\begin{abstract}
L'article fait état des principaux résultats d'une recherche portant sur la réussite scolaire en milieu collégial. Plus spécifiquement, nous y traitons d'un des volets de l'étude, celui de l'influence du système de valeurs des cégépiens sur leur trajectoire scolaire. La recherche a été menée auprès d'un échantillon aléatoire de 744 étudiants de trois cégeps (Gaspésie et des îles, Sainte-Foy, Vieux-Montréal). Un portrait des valeurs des cégépiens en relation avec la réussite scolaire est reproduit. Une comparaison avec d'autres études (québécoises, canadiennes et européennes) est faite au besoin pour mieux contextualiser les résultats de notre recherche. Nous nous appliquons de plus à évaluer l'importance que revêt le système de valeurs des cégépiens pour la réussite scolaire, tant sur le plan du rendement scolaire que sur celui de la persévérance aux études. À cet égard, les valeurs des étudiants figurent parmi les premiers déterminants de la réussite scolaire selon nos travaux. Enfin, l'article propose quelques paramètres d'intervention visant à favoriser la réussite scolaire. Ces paramètres ont été conçus sur la base des résultats de la recherche et ils incorporent des facteurs sociaux tenant à la réalité des cégépiens dont, au premier chef, leur système de valeurs.
\end{abstract}

Mots-clés: valeurs, cégépiens, réussite scolaire, cégep, paramètres d'intervention.

This paper presents the main results of a research about successfully completing college studies. More specifically, we consider one of the aspects of the study: the influence of college students' values on their academic evolution. The research was conducted with a random sample of 744 students from three different CEGEPS. A portrait of college students' values in relation with their successful completion of studies is reproduced. A comparison with other research papers (Québécois, Canadian and European) was carried out at the same time to better contextualize the results of our research. Also, this paper intends to evaluate the importance of college students' values on successfully completing their studies, both regarding academic achievement and persistence in school. According to our research, students' values are among the most decisive aspects for successfully completing college 
studies. Finally, this paper suggests some intervention parameters that aim to promote successful completion of college studies. These parameters were conceived based on the research results and they include social factors related to the college students' reality, especially to their values.

Keywords: Values, College students, Successful completion of studies, CEGEP, Intervention parameters.

\section{INTRODUCTION}

Au cours des deux dernières décennies en particulier, la réussite scolaire s'est progressivement imposée comme enjeu social au Québec. Sur le plan institutionnel, dans le réseau de l'éducation, les cégeps sont tenus de soumettre au ministre de l'Éducation un plan de mesures visant à favoriser la réussite localement. Du côté de la communauté scientifique, on observe un certain déplacement des objets de recherche vers des facteurs sociaux extérieurs aux institutions d'enseignement. Selon les écrits scientifiques, ces facteurs conditionneraient le cheminement scolaire des étudiants, de concert avec les traditionnels facteurs endogènes au milieu de l'éducation (types de pédagogies, mesures de rattrapage, qualité de l'environnement éducatif, exigences selon les cycles d'enseignement...). C'est ainsi que des thèmes tels que, par exemple, la conciliation travail-études, les rapports sociaux et familiaux, l'origine sociale des étudiants, leur statut socioéconomique, leur bien-être personnel, leur système de valeurs et les différenciations selon le sexe des étudiants, en lien avec la réussite scolaire, sont de plus en plus sujets d'analyse et d'exploration dans les publications scientifiques.

Dans la foulée de ces préoccupations, une récente recherche intitulée Étude comparée sur la réussite scolaire en milieu collégial selon une approche d'écologie sociale (Roy, Mainguy, Gauthier et Giroux, 2005) rend compte de l'influence de certains facteurs sociaux sur le parcours scolaire des cégépiens.

L'objet du présent article est de rapporter les résultats de la recherche qui ont trait spécifiquement à la contribution du système de valeurs des étudiants à leur réussite scolaire. De fait, dans l'étude, les valeurs des cégépiens ont exercé un rôle important sur leur trajectoire scolaire; de tous les facteurs sociaux que nous avons examinés, le champ des valeurs est celui qui a présenté les résultats les plus significatifs au regard de la réussite scolaire. Les valeurs des étudiants ont aussi constitué un terreau fertile quant à la réflexion sur les avenues d'intervention à développer pour favoriser la réussite. À cet égard, nous présenterons quelques paramètres d'intervention que nous ont suggérés à la fois les constats de la recherche et des discussions que nous avons engagées avec des intervenants en milieu collégial sur le sujet à partir des résultats de l'étude. 


\section{CONTEXTE DE LA RECHERCHE}

La recherche a été réalisée auprès d'un échantillon représentatif et aléatoire de 744 étudiants répartis dans trois collèges d'enseignement général et professionnel (cégep) situés dans des milieux sociologiques distincts : le cégep de la Gaspésie et des îles, le cégep de Sainte-Foy et le cégep du Vieux-Montréal. L'étude vise à comprendre et à expliquer les facteurs sociaux qui conditionnent la réussite scolaire et leurs interrelations selon une approche d'écologie sociale et dans une perspective d'intervention en milieu collégial (Roy et al., 2005, p. 14).

Plus globalement, nous avons interrogé la réussite scolaire en milieu collégial sous l'angle suivant: dans quelle mesure le rapport entre le jeune cégépien et la société (au sens de lien social) exerce-t-il une influence sur le parcours scolaire de l'étudiant? Cette question nous a interpellés principalement du fait que les plans de réussite dans les cégeps sont essentiellement axés sur les facteurs endogènes au réseau de l'éducation, occultant une bonne partie de la problématique de la réussite scolaire qui tient aux facteurs sociaux extérieurs au milieu de l'éducation.

Afin d'examiner la réussite scolaire dans un tel cadre, nous avons donc retenu le modèle de l'écologie sociale comme assise théorique pour mieux traduire le rapport entre l'étudiant et la société, de même que l'impact de ce rapport sur son cheminement scolaire. Précisons que ce modèle s'applique à examiner les liens entre un individu et les différents environnements dans lesquels il évolue.

En ce qui concerne les valeurs des étudiants, elles relèvent, dans le modèle écologique, de l'ontosystème qui renvoie aux caractéristiques personnelles de l'étudiant, tant innées qu'acquises (Bouchard, 1987). Cependant, le système de valeurs des cégépiens n'est pas sans contenir les diverses formes d'influence et de socialisation qui ont pu façonner leurs valeurs et qui puisent aux différents environnements sociaux (famille, réseau d'amis, cégep, milieu de travail, communauté locale, société...). C'est dans cette acception que nous avons porté notre regard sur la problématique des valeurs en lien avec la réussite scolaire.

\section{ÉLÉMENTS DE MÉTHODE}

La recherche comporte deux volets : un volet quantitatif réalisé la première année de l'étude et un volet qualitatif effectué la seconde année à partir d'entrevues de groupe auprès d'un échantillon limité d'étudiants. Aux fins de l'article, nous examinerons essentiellement les résultats de la partie quantitative de l'étude.

Le volet quantitatif de l'étude s'est établi sur la base d'un questionnaire d'enquête administré auprès d'un échantillon systématique probabiliste (degré de confiance de $95 \%$ ) dans chacun des trois cégeps participants. L'étude est de type transversal, c'est-à-dire que les étudiants ont rempli une seule fois le questionnaire, à la fin de l'automne 2003. 
Le questionnaire d'enquête a été conçu pour répondre aux critères du modèle d'écologie sociale retenu. II se subdivise en six sections :

1. Les caractéristiques personnelles des étudiants (âge, sexe, programme d'études, résultats scolaires...);

2. Le cégep comme milieu de vie (liens avec les professeurs, avec les autres étudiants, temps accordé aux travaux scolaires, participation à des activités parascolaires...);

3. Les réseaux familial et social (liens avec les parents, avec les amis, soutien parental, bénévolat dans la communauté...);

4. Le bien-être personnel (indicateurs de santé mentale, tels que le stress, l'estime de soi, la consommation d'alcool ou de drogues...);

5. Le système de valeurs (défini sur la base de propositions d'énoncés couvrant un éventail varié de valeurs et d'aspirations);

6. La situation socioéconomique (participation au marché du travail, situation financière, prêts et bourses du ministère de l'Éducation du Québec...).

Les informations sur ces sections ont été mises en relation entre elles, d'une part, et avec les indicateurs de réussite scolaire, d'autre part, conformément à la perspective d'écologie sociale que nous avons adoptée.

Dans le plan d'analyse, deux variables clés ont servi à qualifier la réussite scolaire de l'étudiant: les résultats exprimant le rendement scolaire et la persévérance aux études. Cette dernière information a été évaluée à partir d'une question demandant aux étudiants s'ils songeaient à abandonner leurs études. Ces variables « dépendantes » ont été mises en relation avec l'ensemble des autres variables de l'étude. Précisons que le rendement scolaire et la persévérance dans les études sont deux dimensions complémentaires de la réussite. Parfois, elles s'entrecroisent pour former des entrelacs, parfois elles s'éloignent, mettant en présence des facteurs associés à la réussite qui sont différents. Elles conduisent aussi à des stratégies d'intervention et à des actions qui ne sont pas toujours de même nature.

Diverses analyses statistiques ont été réalisées, notamment le test du « chi carré » pour apprécier le degré d'association des valeurs avec les autres variables du questionnaire d'enquête, une analyse factorielle pour regrouper des valeurs sous des thèmes généraux et des analyses multivariées (régressions multiples avec procédure de sélection des variables) pour dégager certaines combinaisons de valeurs permettant de prédire le rendement scolaire des cégépiens.

La répartition des étudiants dans l'échantillon (744 étudiants) traduit bien leur représentation selon la taille du cégep, selon le sexe (65\% féminin, $35 \%$ masculin) et selon le secteur d'études (54\% au secteur technique, $46 \%$ au secteur préuniversitaire). 
Ces étudiants avaient des résultats comparables à ceux de l'ensemble des élèves (moyenne cumulative de $74 \%$ pour notre échantillon et de 73,3\% pour l'ensemble des étudiants). Quant à la question de l'abandon scolaire (mesure de la persévérance scolaire), soulignons que $16 \%$ des élèves de la recherche songeaient, «à l'occasion » ou « sérieusement », à abandonner leurs études; $84 \%$ n'y pensaient «pas du tout ».

En ce qui concerne les valeurs, nous avons retenu la définition suivante de Bréchon :

Les valeurs sont des idéaux, des préférences qui prédisposent les individus à agir dans un sens déterminé. Elles appartiennent aux orientations profondes qui structurent les représentations et les actions d'un individu [...]. Les valeurs d'un individu constituent son identité profonde, ce qui le mobilise et le fait vivre (2000, p. 9).

Sur cette base et à partir des écrits scientifiques existants, nous avons eu recours à la formule d'énoncés de valeurs où les étudiants avaient à marquer leur préférence. Par la suite, les résultats ont été mis en relation avec les indicateurs de la réussite scolaire et les autres variables du questionnaire d'enquête.

\section{BREF PARCOURS DE LA LITTÉRATURE}

Les études comme les sondages sur les valeurs des jeunes sont légion. Nous avons retenu ici les ouvrages qui nous sont apparus les plus significatifs dans le contexte de notre recherche, c'est-à-dire des travaux qui ont exploré les valeurs selon certaines dimensions directement liées à la problématique de la réussite scolaire.

Un récent ouvrage collectif intitulé Les valeurs des jeunes, sous la direction de G. Pronovost et de C. Royer (2004), rassemble des textes de différents auteurs qui font le point sur l'évolution des valeurs des jeunes.

Mentionnons entre autres un texte (Royer, Pronovost et Charbonneau, 2004) qui met en lumière le fait que les études soient perçues, dans l'esprit des jeunes, selon une conception utilitariste, c'est-à-dire que ce ne serait pas tant le savoir en tant que tel qu'on valoriserait chez les étudiants, mais bien « ce que donnent les études » en lien avec l'emploi, la carrière, la réussite sociale. Le collectif de Pronovost et Royer (2004) s'inscrit dans la liste des ouvrages généraux sur la question des valeurs des jeunes avec notamment certaines études françaises relativement connues, dont celles de Bréchon (2000), de Galland et Roudet (2001) et de Boudon (2002).

Certains travaux ont abordé des dimensions spécifiques. Il en est ainsi de l'ouvrage de Rivière et Jacques (2002) qui a porté sur la relation sociale des cégépiens au regard de la réussite. Les auteurs évoquent notamment l'existence de différences parfois sensibles entre filles et garçons quant à leur représentation de la réussite. 
C'est ainsi que les garçons auraient davantage de croyances qui les pousseraient au «dilettantisme » face aux études, où le désintérêt se ferait sentir lorsque le cégep n'est pas synonyme de plaisir chez eux. Pour les filles, les études constitueraient l'aboutissement d'un projet personnel, ce qui expliquerait une motivation plus forte chez elles; elles croiraient davantage à l'effort pour réussir et les camarades de classe seraient davantage une source d'appui pour les études, alors qu'ils seraient plutôt prétexte à compétition chez les garçons (Rivière et Jacques, 2002).

Les écrits scientifiques sur les valeurs parus depuis les années 1980 (Attias-Donfut, 2002; Bernier, 1986; Bernier et Gauthier, 1997; Bibby et Posterski, 1986; Cournoyer, 1985; Lazure, 1986; Paquette, 1996; Royer, Pronovost et Charbonneau, 2004) révèlent une tendance lourde qui est encore bien actuelle : les liens socioaffectifs de base (les amis, la famille) sont une priorité dans l'échelle de valeurs des jeunes. De récents sondages au Québec (Crop, 2002, 2004) ont confirmé cet état de fait. Dans nos propres travaux (Roy et al., 2003; Roy, 2004, 2006), nous avons pu constater à quel point la famille revêt une importance singulière dans l'esprit des cégépiens - même si, en même temps, à cet âge, nous observons un processus de distanciation de la famille lié à une quête d'autonomie.

Ces quelques mentions bibliographiques nous serviront de points de repère dans l'analyse des résultats de notre étude.

\section{LES VALEURS DES CÉGÉPIENS ET LA RÉUSSITE SCOLAIRE}

L'exploration des valeurs chez les étudiants nous a permis de mieux connaître ces derniers et de dégager un portrait qui rompt parfois de façon marquée avec les représentations populaires et les discours ambiants à leur sujet.

En premier lieu, un constat qui nous accompagne tout au long de l'étude: dans l'échelle de valeurs des cégépiens, réussir ses études, c'est très important. De fait, huit étudiants sur dix (80 \%) considèrent que c'est « très important », $18 \%$ estiment que c'est « important », alors que $2 \%$ accordent peu d'importance à la réussite des études. Malgré le fait que les filles accordent généralement davantage d'importance aux études $(P=0,000)$, il n'en reste pas moins que filles comme garçons semblent bien engagés sur le plan des valeurs concernant la réussite des études. Ainsi, $83 \%$ des filles et $72 \%$ des garçons considèrent que réussir ses études, c'est « très important ». Cette variable - l'importance accordée à la réussite des études - est apparue comme l'une des plus révélatrices parmi celles qui sont associées à la réussite scolaire.

La presque totalité des étudiants $(97 \%)$ ont souligné que, pour leur famille, la poursuite des études est une valeur « importante » (dont $61 \%$ ont signifié qu'elle était « très importante »). En corollaire, nous avons documenté l'existence de formes de solidarités familiales se traduisant par un appui moral et financier tangible des parents aux jeunes cégépiens afin de favoriser leur parcours scolaire. L'importance 
que revêt la dimension « familiale » dans l'esprit des étudiants se traduit notamment dans le prochain tableau. Celui-ci nous donne les résultats d'un exercice où nous avons demandé aux étudiants de se projeter dans l'avenir et de nous révéler lequel des énoncés que nous retrouvons au tableau 1 correspond le mieux à la représentation qu'ils se font d'une vie « réussie ».

Tableau 1

Indice déterminant l'importance accordée par les étudiants des trois cégeps à cinq énoncés portant sur des aspirations de vie

\begin{tabular}{|c|l|c|}
\hline \multirow{2}{*}{ Rang } & \multicolumn{1}{|c|}{$\begin{array}{c}\text { Énoncés } \\
\text { Plus tard, tu penseras avoir réussi ta vie si : }\end{array}$} & Indice $^{\mathbf{1}}$ \\
\hline \hline $1^{\mathrm{er}}$ & Tu as une famille unie. & 3,74 \\
\hline $2^{\mathrm{e}}$ & Tu obtiens du succès dans ton travail. & 3,47 \\
\hline $3^{\mathrm{e}}$ & Tu t'engages dans ton milieu. & 2,72 \\
\hline $4^{\mathrm{e}}$ & Tu fais beaucoup d'argent. & 2,66 \\
\hline $5^{\mathrm{e}}$ & Tu es important (importante) et influent (influente). & 2,41 \\
\hline
\end{tabular}

Tant chez les filles que chez les garçons, la famille et le travail constituent des valeurs premières quand ils se projettent dans l'avenir. En ce qui a trait à la famille spécifiquement, on peut observer que cette préférence accordée à la dimension «famille » fait écho à bon nombre de recherches qui soulignent l'importance de cette valeur dans l'esprit des jeunes, voire sa progression depuis les deux dernières décennies en particulier (Attias-Donfut, 2002; Bernier, 1997; Crop, 2002; Crop, 2004; Galland et Roudet, 2001; Pronovost et Royer, 2004; Roy, 2004, 2006).

Sur un registre plus large, nous avons sondé les jeunes cégépiens sur un éventail varié de valeurs à partir des énoncés contenus au tableau 2. Celui-ci reflète des tendances que nous aurons l'occasion, plus loin, de discuter et de mettre en relation avec la réussite scolaire. Soulignons que, tout comme dans le tableau 1, nous n'observons pas de différences notables entre les cégeps.

1. Les étudiants avaient à qualifier chacun des énoncés à partir d'une échelle variant de 1 (le moins important) à 5 (le plus important). L'indice reproduit la moyenne obtenue pour chacun des énoncés. 
Tableau 2

Importance relative des valeurs des élèves des trois cégeps sur la base d'énoncés portant sur des valeurs

\begin{tabular}{|c|l|c|}
\hline Rang & \multicolumn{1}{|c|}{ Énoncés } & Indice \\
\hline $1^{\text {er }}$ & Respecter l'autre dans sa différence est important pour moi. & 3,76 \\
\hline $2^{\mathrm{e}}$ & Acquérir des connaissances est important pour moi. & 3,75 \\
\hline $3^{\mathrm{e}}$ & Devenir compétent (compétente) sur le plan professionnel m'apparaît important. & 3,74 \\
\hline $4^{\mathrm{e}}$ & Je crois à l'importance de l'effort pour réussir dans mes études. & 3,71 \\
\hline $5^{\mathrm{e}}$ & Il est important pour moi d'être autonome. & 3,68 \\
\hline $6^{\mathrm{e}}$ & Bien faire les choses que j'entreprends est important pour moi. & 3,65 \\
\hline $7^{\mathrm{e}}$ & Il est important de se dépasser dans ce que l'on fait. & 3,60 \\
\hline $8^{\mathrm{e}}$ & La famille est une dimension importante dans ma vie. & 3,58 \\
\hline $9^{\mathrm{e}}$ & Posséder une bonne culture générale est important pour moi. & 3,54 \\
\hline $10^{\mathrm{e}}$ & Coopérer avec les autres est important pour moi. & 3,49 \\
\hline $11^{\mathrm{e}}$ & Le diplôme collégial a une signification pour moi. & 3,47 \\
\hline $12^{\mathrm{e}}$ & Avoir des projets à long terme est important pour moi. & 3,35 \\
\hline $13^{\mathrm{e}}$ & Ce qui compte, c'est le plaisir. & 3,27 \\
\hline $14^{\mathrm{e}}$ & Ce qui compte, c'est d'être bien apprécié (appréciée) par son milieu. & 3,19 \\
\hline $15^{\mathrm{e}}$ & Ce qui compte, c'est le présent. & 3,11 \\
\hline $16^{\mathrm{e}}$ & L'apparence est quelque chose d'important pour moi. & 2,76 \\
\hline $17^{\mathrm{e}}$ & Il est important d'être compétitif ou compétitive dans la vie. & 2,56 \\
\hline $18^{\mathrm{e}}$ & La consommation de biens matériels est importante pour moi. & 2,49 \\
\hline $19^{\mathrm{e}}$ & Les valeurs spirituelles sont une dimension importante dans ma vie. & 2,40 \\
\hline $20^{\mathrm{e}}$ & Ce qui compte, c'est de gagner rapidement de l'argent. & 2,07 \\
\hline
\end{tabular}

Sommairement, deux conclusions se dégagent de ce tableau. La première consiste à prendre acte de la prégnance de valeurs qui s'accordent avec le monde de l'éducation chez les étudiants. Et ce, tant chez les filles que chez les garçons même si, par ailleurs, « l'intensité » enregistrée pour ces valeurs est plus élevée chez les étudiantes. Des valeurs telles que l'acquisition de connaissances, l'aspiration liée à la compétence professionnelle ou la «méritocratie » (récompense pour les efforts fournis pendant les études) figurent au haut de la liste des énoncés retenus par les étudiants. Ces mêmes valeurs sont associées positivement au rendement scolaire comme à la persévérance aux études, ce que nous examinerons plus loin.

2. Les étudiants avaient à évaluer leur degré d'accord pour chacun des énoncés selon la légende suivante : 4- tout à fait d'accord; 3- plutôt en accord; 2- plutôt en désaccord; 1- tout à fait en désaccord. L'indice reproduit la moyenne. 
La seconde conclusion vient casser le mythe voulant que les valeurs matérielles surclassent celles qui sont liées au savoir dans l'esprit des jeunes cégépiens. Selon nos résultats, « l'apparence », la consommation de biens matériels ou le gain rapide d'argent sont des valeurs logeant au bas du tableau (même si, par ailleurs, elles ne sont pas sans traduire un mode de vie chez plusieurs cégépiens). Ce qui apparaît positif, puisque ces énoncés de valeurs sont généralement liés négativement à la réussite scolaire. Ce double constat - les valeurs liées à l'éducation sont privilégiées et celles associées à la «matérialité » sont plutôt secondarisées - traduit l'existence d'une forme d'engagement parfois sous-estimée des jeunes à l'égard des études.

Le caractère volontairement "éclaté » des énoncés de valeurs proposés aux étudiants afin de dégager un profil d'ensemble n'exclut pas une parenté entre énoncés qui nous conduit à certains regroupements significatifs. Après avoir procédé à une analyse factorielle, nous avons vu quatre regroupements de variables s'imposer à nous. Nous leur avons assigné un thème pour les désigner globalement.

Tableau 3

Regroupement des valeurs par thèmes

\begin{tabular}{|llc|}
\hline $\begin{array}{l}\text { Thèmes } \\
\text { alpha }\end{array}$ & \multicolumn{1}{c|}{ Valeurs } & Coefficient \\
\hline La réussite & $\begin{array}{l}\text { Importance accordée aux études. } \\
\text { Importance accordée au dépassement dans ce que l'on fait. } \\
\text { Importance accordée à l'obtention du diplôme collégial. } \\
\text { Importance accordée à la nécessité de bien faire les choses } \\
\text { que l'on entreprend. } \\
\text { Importance accordée aux projets à long terme. } \\
\end{array} \quad \begin{array}{l}\text { Importance accordée à l'effort pour réussir ses études. } \\
\text { Importance accordée à l'acquisition de compétences } \\
\text { sur le plan professionnel. }\end{array}$ & \\
\hline La matérialité & $\begin{array}{l}\text { Importance accordée à l'apparence. } \\
\text { Importance accordée à la consommation de biens matériels. } \\
\text { Importance accordée au fait de gagner rapidement de l'argent. }\end{array}$ & $\mathbf{0 , 6 6}$ \\
\hline La culture & $\begin{array}{l}\text { Importance accordée à l'acquisition de connaissances. } \\
\text { Importance accordée à l'acquisition d'une bonne culture générale. }\end{array}$ & $\mathbf{0 , 5 8}$ \\
\hline L'intégration & $\begin{array}{l}\text { Importance accordée au respect de l'autre dans sa différence. } \\
\text { Importance accordée à la coopération. }\end{array}$ & $\mathbf{0 , 5 5}$ \\
& $\begin{array}{l}\text { Importance accordée à la famille. } \\
\text { Importance accordée à la spiritualité. }\end{array}$ & \\
\hline
\end{tabular}


Dans chacun de ces regroupements, les variables sont étroitement associées entre elles. Elles forment une sorte de logique interne, de famille de variables qui nous ont parfois été utiles pour apprécier certaines tendances.

Le thème de la « réussite » parle par lui-même. II présente un maillage de variables qui convergent toutes - à des degrés divers - vers la réussite scolaire. Celui de la " culture » se pose en complément à la réussite. Il a par ailleurs sa propre cohérence interne. Le thème de la «matérialité » procède d'une logique qui lui est propre également et qui, de façon générale, selon nos résultats, éloigne de la réussite, ainsi que nous l'avons évoqué plus haut.

Le thème de «l'intégration », contrairement aux trois premiers, n'offre pas spontanément une lecture commune; le fil d'Ariane est moins visible. S'il existe un point d'ancrage entre les quatre variables de ce regroupement, il réside, croyons-nous, dans le concept de « logique de l'intégration » au sens que Bajoit (2003) y prête, à savoir une recherche de stabilité. Ainsi, les liens entre famille et spiritualité (valeurs de stabilité) sont les plus éloquents dans les travaux de Bajoit comme dans les nôtres où viennent se greffer des valeurs liées à la coopération avec les autres (manière de renforcer l'intégration au cégep?) et à la tolérance (manière de s'intégrer en assumant le respect des autres, ce qui favoriserait l'ouverture et, éventuellement, la coopération?). Ce sont là des hypothèses exploratoires. Les quatre regroupements d'énoncés de valeurs constituent davantage, pour le moment, des constructions statistiques qui mériteraient ultérieurement un examen plus attentif.

À l'échelle des valeurs, c'est du côté du sexe des étudiants que l'on repère des différences qui comptent. Ces différences se résument de la façon qui suit.

- Les filles accordent plus d'importance que les garçons :

- aux études

$$
\begin{aligned}
& P=0,000 \\
& P=0,000 \\
& P=0,000 \\
& P=0,000 \\
& P=0,000 \\
& P=0,000 \\
& P=0,000 \\
& P=0,000
\end{aligned}
$$

- à l'effort pour réussir leurs études

- à l'obtention du diplôme collégial

- à l'autonomie

- à la coopération avec les autres

- à la nécessité de bien faire les choses

- au respect de l'autre

- à la famille

- Les garçons accordent plus d'importance que les filles :

- à la compétition

$P=0,000$

- au fait de gagner rapidement de l'argent

$\mathrm{P}=0,000$

- au plaisir

$P=0,004$

- au temps présent

$P=0,011$ 
Ces différences méritent qu'on s'y attarde quelque peu. En premier lieu, il faut dissiper une illusion que pourraient créer les différences observées selon le sexe des étudiants, à savoir que les valeurs des garçons sont éloignées de celles recherchées dans le milieu de l'éducation. Une brève analyse nous fait voir au contraire qu'il existe une parenté « structurelle » quant à l'importance accordée aux énoncés de valeurs entre les filles et les garçons. C'est ainsi que les cinq premiers énoncés de valeurs qui figurent au tableau 2 sont les mêmes chez les deux sexes, bien que l'ordre diffère quelque peu. De la même manière, les sept derniers sont également les mêmes (l'ordre par rang est relativement similaire ici).

Cela signifie que, globalement, filles comme garçons ont un système de valeurs qui sert de point d'appui à la réussite scolaire. Cependant, à la marge, on enregistre des différences selon le sexe des étudiants et ces différences, telles qu'elles sont rapportées plus haut, jettent un nouvel éclairage sur la réussite sous l'angle des valeurs. Ainsi, on observe que les filles ont un système de valeurs qui traduit d'une manière affermie une «culture de la réussite ». De fait, des valeurs comme l'importance accordée aux études, à l'effort pour réussir, au diplôme collégial, à l'autonomie, à bien faire les choses, à la coopération avec d'autres - valeurs davantage privilégiées chez les filles - sont autant de déterminants de la réussite scolaire.

Cette «culture de la réussite », définie ici sur la base d'un repérage d'énoncés de valeurs associées à la réussite, est présente chez les garçons, mais elle est plus accentuée chez les filles. À l'inverse, des valeurs comme l'importance accordée au fait de gagner rapidement de l'argent ou comme celle accordée au temps présent (culture de l'immédiateté) sont davantage présentes chez les garçons et, selon nos résultats, associées négativement à la réussite scolaire. Encore une fois, répétonsle, c'est à la marge (soit essentiellement auprès des étudiants à risque d'échec ou d'abandon scolaire) que ces considérations vont exercer leur influence selon le sexe des étudiants. Ces résultats ouvrent une piste de réflexion autour de la question des valeurs comme source d'explication - partielle, soulignons-le - d'une réussite scolaire plus manifeste chez les filles selon les statistiques existantes.

Sur le terrain spécifique de la réussite scolaire, les valeurs comptent parmi les déterminants importants de la trajectoire scolaire des cégépiens. Le tableau suivant reproduit la meilleure combinaison de valeurs au regard de la réussite. C'est-à-dire que les étudiants qui cumulent ces valeurs affichent un meilleur rendement scolaire selon nos résultats. 


\section{Tableau 4 \\ Meilleure combinaison des valeurs prédictrices du rendement scolaire pour les élèves des trois cégeps}

\begin{tabular}{|l|c|}
\hline \multicolumn{1}{|c|}{ Variables prédictrices } & $\begin{array}{c}\text { r' Pourcentage } \\
\text { cumulatif de la } \\
\text { variance expliquée } \\
\text { (\%) }\end{array}$ \\
\hline \hline L'étudiant ou l'étudiante qui a de bons résultats scolaires : & $\mathbf{6}$ \\
\hline 1) accorde de l'importance aux études; & $\mathbf{8}$ \\
\hline 2) trouve moins important de gagner rapidement de l'argent; & $\mathbf{9}$ \\
\hline 3) accorde de l'importance à l'acquisition de compétences sur le plan professionnel; & $\mathbf{1 0}$ \\
\hline 4) trouve important de bien faire les choses; & $\mathbf{1 1}$ \\
\hline 5) accorde moins d'importance à l'apparence; & $\mathbf{1 2}$ \\
\hline 6) accorde de l'importance à la coopération avec les autres; & $\mathbf{1 2}{ }^{3}$ \\
\hline 7) accorde moins d'importance à la consommation de biens matériels. & \\
\hline
\end{tabular}

La variable clé qui nous accompagne ici est liée à l'importance accordée aux études dans les valeurs personnelles de l'étudiant, et cela, tant chez les filles que chez les garçons! C'est notre variable « phare » sur le plan des valeurs. Les autres résultats du tableau 4 mettent en évidence la logique des thèmes sur les valeurs développés plus haut. Ainsi, les valeurs qui se regroupent autour du thème de la « réussite » (importance accordée aux études, à l'acquisition de compétences sur le plan professionnel, à bien faire les choses) sont, par essence, prédictrices de meilleurs résultats scolaires. Les valeurs liées au thème de la "matérialité » (importance accordée au gain rapide d'argent, à l'apparence et à la consommation de biens matériels) sont négativement associées à la réussite.

En procédant aux mêmes analyses, cette fois-ci en distinguant selon le sexe des étudiants, on retrouve la synthèse suivante :

\begin{tabular}{|c|c|}
\hline Filles & Garçons \\
\hline 1) Accordent de l'importance à l'effort pour réussir. & 1) Accordent de l'importance aux études. \\
\hline 2) Trouvent important de bien faire les choses. & 2) Accordent moins d'importance au plaisir. \\
\hline $\begin{array}{l}\text { 3) Accordent de l'importance à la coopération avec les } \\
\text { autres. }\end{array}$ & $\begin{array}{l}\text { 3) Trouvent important de devenir compétents sur le } \\
\text { plan professionnel. }\end{array}$ \\
\hline
\end{tabular}

3. Pour l'item 6 , la valeur de prédiction est de $11,7 \%$, alors que pour l'item 7 elle est de $12,2 \%$. Aux fins de présentation du tableau, nous avons arrondi à 12 pour l'item 6 . 
L'effort et l'application distinguent le plus souvent les filles des garçons à l'échelle des valeurs associées à la réussite scolaire. Ce constat permet d'expliquer, en partie, pourquoi la variable «difficultés scolaires » figure au premier rang des variables prédisant l'abandon scolaire chez les filles, alors qu'à ce titre le manque d'intérêt pour les études joue le même rôle chez les garçons. On peut proposer l'interprétation suivante: de façon générale, malgré les difficultés scolaires, les filles, du fait de leur système de valeurs qui privilégie l'effort, vont davantage persévérer, parfois jusqu'au seuil de rupture des études. Elles adhèrent à l'idéologie de la « méritocratie », enfin, plus que les garçons dans l'ensemble.

\section{DES PISTES POUR L'INTERVENTION}

Au terme de notre recherche, nous avons engagé une démarche en vue de définir des paramètres d'intervention sur la base du matériel de l'étude. Ces paramètres visent à fournir un cadre de réflexion pour les cégeps désireux d'intégrer la logique des facteurs sociaux dans leur plan de réussite.

À cet effet, nous avons puisé à trois sources. Une première porte sur des écrits professionnels et scientifiques existants concernant les interventions. Une seconde réside dans le matériel d'entrevues de groupe que nous avons recueilli dans le volet qualitatif de la recherche menée auprès d'une soixantaine d'étudiants (35 filles, 29 garçons), sélectionnés au hasard dans les trois cégeps participants de l'étude, relativement aux interventions qu'ils souhaitent pour améliorer la réussite. Ce matériel a été obtenu sur la base d'un schéma d'entrevue semi-dirigée dans le volet qualitatif de l'enquête. Enfin, nous avons rencontré des intervenants (une vingtaine en tout) à l'occasion d'entrevues de groupe dans chacun des trois cégeps pour partager avec eux une réflexion sur les actions à conduire à partir des constats de la recherche. Nous discuterons des paramètres qui concernent plus directement le champ des valeurs des cégépiens.

Un premier paramètre d'intervention est lié à la culture du cégep, lieu d'éducation et de socialisation. Ainsi, dans la société, il existe une panoplie de discours qui dévalorisent le savoir tout en exerçant une influence certaine sur le rapport qu'entretiennent les jeunes cégépiens à l'égard de leurs études. Bon nombre d'auteurs ont relevé ce fait. Une auteure résume à sa manière ce point de vue : "Quant à l'éducation, il est à se demander si les valeurs marchandes véhiculées par le néolibéralisme n'ont pas fini par débrancher les jeunes, pressés de faire un choix entre l'argent, la consommation et le savoir » (Nduwimana, 2003, p. B-5).

Des intervenants rencontrés en entrevues de groupe nous ont fait valoir l'importance pour le cégep d'affirmer bien haut et fort ses valeurs et de faire la promotion du savoir en se posant en alternative aux discours ambiants discréditant le savoir et la culture. Ainsi, des actions visant à consolider l'existence d'une culture commune au cégep permettraient d'agir auprès de certains étudiants peu motivés pour les études et davantage influencés par les valeurs qui les éloignent du collège. 
L'éventail des moyens à définir sur le plan local à cet égard est relativement large. Cependant, la contribution du secteur parascolaire est apparue des plus indiquées pour intervenir notamment sur le renforcement identitaire de l'étudiant par rapport à son programme d'études et sur l'ouverture au monde et à la société dans une perspective de développement personnel à la citoyenneté.

Un second paramètre d'intervention a trait à la conciliation travail-études. Nos travaux révèlent que sept étudiants sur dix occupent un emploi pendant l'année scolaire. Filles comme garçons! Au-delà d'un certain seuil d'heures accordées au travail rémunéré (environ 25 heures par semaine, selon nos résultats), les risques d'échec et d'abandon scolaires surgissent. Des interventions visant à sensibiliser les employeurs à la réalité des étudiants et à établir sur une base régionale des conditions minimales - par l'entremise de tables de concertation socioéconomique pour éviter toute forme «d'exploitation » de la main-d'œuvre étudiante - ont été évoquées. D'autres interventions s'adressant directement à l'étudiant à travers des activités de tutorat, par exemple, afin qu'il puisse opérer des choix adéquats entre le travail, les études et ses autres activités ont également été signalées par les participants. Ce serait alors un moment jugé privilégié selon eux pour confronter les valeurs des étudiants au regard de leurs aspirations scolaires et professionnelles.

Un troisième paramètre concerne la relation entre le professeur et l'étudiant. L'étude a mis en évidence que la qualité de cette relation comptait parmi les déterminants de la réussite scolaire (Roy et al., 2005, p. 61-63). Selon le point de vue des étudiants, l'amélioration de la relation entre eux et les professeurs, tant sur le plan relationnel que pédagogique, ne peut que favoriser la réussite. Les cégépiens suggèrent d'instaurer un mécanisme d'évaluation des enseignants. Dans leur esprit, ce mécanisme d'évaluation fournirait aux professeurs les indications nécessaires pour qu'ils puissent rajuster le tir et progresser.

De fait, bon nombre d'enseignants rencontrés ont fait part de leurs difficultés à lire la mutation des valeurs qui s'opère chez les nouvelles générations. Celles-ci seraient moins idéologiques et davantage pragmatiques selon Boudon (2002). Pour Boudon encore, les jeunes d'aujourd'hui seraient imprégnés d'une forme de « rationalité instrumentale » qui les conduit à interroger par exemple la matière du cours selon des critères utilitaires. Également, les étudiants adopteraient une position plus critique qu'auparavant face à l'autorité (pour eux, ce n'est pas le statut de professeur qui est garant de la "vérité » dans les cours) et ils désirent davantage participer en classe selon divers moyens pédagogiques qui favorisent la réappropriation des savoirs. Ces seuls aspects que nous venons de mentionner constituent un choc culturel pour certains enseignants. 


\section{CONCLUSION}

Tout au long de notre parcours, nous avons constaté l'importance que revêt le système de valeurs des jeunes en lien avec la réussite scolaire. Les valeurs des cégépiens agissent comme déterminants de premier plan de leur trajectoire scolaire. Filles comme garçons!

D'une certaine manière, les jeunes forment un microcosme de l'évolution des sociétés. Ils témoignent de certaines métamorphoses sociales qui ont pu s'opérer dans le temps. II en est ainsi, par exemple, du pragmatisme qui les caractérise et de leur mode de vie qui trahit une vision productiviste de l'existence. Une illustration : la semaine de 60 heures pour un étudiant sur cinq, selon notre étude.

Le détour des valeurs apparaît incontournable sur le plan de l'intervention. Voilà ce qui est également ressorti comme constante dans l'étude. À cet égard, les mises en chantier sont multiples. Cependant, comme nous venons de l'évoquer, les transformations sociales qui se succèdent à un rythme de plus en plus rapide nous commandent des «mises à jour » régulières du portrait des jeunes pour mieux réfléchir sur la réussite scolaire comme sur d'autres aspects de leur réalité sociale. C'est la raison pour laquelle nous résistons à l'idée que les constats observés dans nos travaux expriment des tendances lourdes pour demain. Ils pourraient néanmoins nous accompagner pour quelques années encore.

En complément à cet article, nous publierons dans le prochain numéro de la revue les résultats d'une enquête nationale auprès d'un échantillon de 1729 étudiants concernant leurs valeurs en relation avec la réussite scolaire.

\section{RÉFÉRENCES BIBLIOGRAPHIQUES}

ATtIAS-Donfut, C. (2002). Le nouvel esprit de famille, Paris, Odile Jacob.

BAJoIT, G. (2003). Le changement social. Approche sociologique des sociétés contemporaines, Paris, Armand Colin.

BERNIER, L. (1986). « Tant qu'ils choisissent de vieillir... Point de vue sur les aspirations des jeunes », dans F. Dumont (dir.), Une société des jeunes?, Québec, Les Éditions de I'IQRC, p. 29-44.

Bernier, L. (1997). «Les relations sociales », dans M. GAUthIER et L. Bernier (dir.), Les 15-19 ans. Quel présent? Vers quel avenir?, Québec, Les Presses de l'Université Laval et Les Éditions de l'IQRC, p. 227-236.

Bernier, L., et M. GaUthiER (1997). «Conclusion : entrer dans la vie aujourd'hui », dans M. GAUTHIER et L. BeRnieR (dir.), Les 15-19 ans. Quel présent? Vers quel avenir?, Québec, Les Presses de l'Université Laval et Les Éditions de l'IQRC, p. 227-236.

BiBby, R. et D.C. Posterski (1986). La nouvelle génération: les opinions des jeunes du Canada sur leurs valeurs, Montréal, Les Éditions Fides. 
BOUCHARD, C. (1987). «Intervenir à partir de l'approche écologique: au centre, l'intervenant », Service social, vol. 36, $\mathrm{n}^{\text {os }} 2-3$, p. 455-477.

Boudon, R. (2002). Déclin de la morale? Déclin des valeurs?, Québec, Éditions Nota Bene.

BrÉCHON, P. (dir.) (2000). Les valeurs des Français. Évolution de 1980 à 2000, Paris, Armand Colin.

Cournoyer, M. (1985). Avoir 15 ou 16 ans en 1985 : l'univers culturel des jeunes, Québec, Gouvernement du Québec.

CROP (2002). Résultats d'un sondage d'opinion publié dans Le Soleil le 28 décembre.

CROP (2004). Résultats d'un sondage d'opinion publié dans La Presse, en particulier les 11, 13, 14 et 15 septembre.

GAlland, O., et B. Roudet (dir.) (2001). Les valeurs des jeunes. Tendances en France depuis 20 ans, Paris, L'Harmattan.

LAZURE, J. (1986). «Les modes de vie des jeunes », dans Une société des jeunes?, Québec, Les Éditions de l'IQRC.

NduWimanA, F. (2002). «Le féminisme n'explique pas le problème des gars », Le Devoir, 5 et 6 octobre, p. B-5.

PAquetTe, C. (1996). «Attentes et préoccupations des jeunes de l'époque », Vie pédagogique, $\mathrm{n}^{\circ} 100$, septembre-octobre, p. 15.

Pronovost, G., et C. Royer (dir.) (2004). Les valeurs des jeunes, Québec, Presses de I'Université du Québec.

RIVIĖRE, B., en collaboration avec J. JACQUES (2002). Les jeunes et les représentations sociales de la réussite, Montréal, Éditions Logiques.

Roy, J. (2004). «Valeurs des collégiens et réussite scolaire: convergences et divergences », dans G. Pronovost et C. RoYer (dir.), Les valeurs des jeunes, Québec, Presses de l'Université du Québec, p. 93-109.

Roy, J. (2006). Les logiques sociales et la réussite scolaire des cégépiens, Québec, Les Presses de l'Université Laval et Les Éditions de l'IQRC.

Roy, J., en collaboration avec M. GAUthIER, L. GIROuX et N. MAINGUY (2003). Des logiques sociales qui conditionnent la réussite. Étude exploratoire auprès des étudiants du Cégep de Sainte-Foy. Rapport de recherche PAREA, Sainte-Foy, Cégep de SainteFoy/Observatoire Jeunes et Société.

Roy, J., et N. MAINGUY, en collaboration avec M. GAUTHIER et L. GIROuX (2005). Étude comparée sur la réussite scolaire en milieu collégial selon une approche d'écologie sociale. Rapport de recherche PAREA, Sainte-Foy, Cégep de Sainte-Foy/Observatoire Jeunes et Société.

Royer, C., G. Pronovost et S. Charbonneau (2004). «Valeurs sociales fondamentales de jeunes Québécoises et Québécois. Ce qui compte pour eux », dans G. PRONOvost et C. ROYER (dir.), Les valeurs des jeunes, Québec, Presses de l'Université du Québec, p. 49-69.

TESSIER, R. (dir.) (1989). Pour un paradigme écologique, La Salle, Éditions Hurtubise, HMH. 